\title{
Déficit motor asociado a analgesia epidural en paciente con patología neurológica preexistente no conocida
}

\author{
T. García Navia, M. C. Pérez Mateos, I. Sánchez Martín, L. Olmedo Granados y C. Ávila Gordo \\ Departamento de Anestesiología y Reanimación. Hospital Universitario de Valme. Sevilla
}

García Navia T, Pérez Mateos MC, Sánchez Martín I, Olmedo Granados L, Ávila Gordo C. Déficit motor asociado a analgesia epidural en paciente con patología neurológica preexistente no conocida. Rev Soc Esp Dolor 2013; 20(4): 176-179.

\begin{abstract}
There are many studies that suggest that the neurological complications associated with the practice of neuraxial blockade may have a higher incidence in patients with preexisting neurological conditions such as spinal stenosis. This effect may be particularly relevant if there is no previous diagnosis of these pathologies. We describe our experience with a 60-year-old woman diagnosed with chronic ischemia of the right leg which presented an important motor deficit following epidural catheter insertion for pain management.
\end{abstract}

Key words: Epidural analgesia. Local anesthetic toxicity. Neurological complications. Polyneuropathy.

\section{RESUMEN}

Existen múltiples estudios que afirman que las complicaciones neurológicas asociadas a la práctica de un bloqueo neuroaxial pueden tener una mayor incidencia en los pacientes que presentan enfermedades neurológicas preexistentes como la estenosis espinal. Esta incidencia puede ser especialmente relevante si no se cuenta con un diagnóstico previo de dichas patologías. En el presente trabajo describimos nuestra experiencia con una mujer de 60 años de edad, diagnosticada de isquemia crónica de miembro inferior derecho, que presentó un déficit

Recibido: 03-02-12.

Aceptado: 03-06-12 motor importante tras la colocación de un catéter epidural para el manejo del dolor.

Palabras clave: Analgesia epidural. Toxicidad de anestésicos locales. Complicaciones neurológicas. Polineuropatía.

\section{INTRODUCCIÓN}

La anestesia/analgesia epidural se ha convertido en la técnica neuroaxial de referencia para la analgesia obstétrica y numerosos tipos de cirugías.

La paraplejia y la paraparesia son complicaciones raras asociadas al bloqueo neuroaxial. En varios estudios se afirma que la incidencia de paraparesia secundaria a anestesia epidural oscila entre 0,0005-0,02\% $(1,2)$.

El compromiso de la perfusión de la médula espinal presenta diversas etiologías, pero son dos los principales mecanismos fisiopatológicos implicados: disminución en el flujo sanguíneo (hipotensión prolongada e insuficiencia arterial) y factores mecánicos (hematoma, absceso, metástasis, etc.) (3).

\section{CASO CLÍNICO}

Mujer de 60 años de edad, con peso de $48 \mathrm{~kg}$, talla de $156 \mathrm{~cm}$ y ASA III. Exfumadora de 20 cigarrillos diarios, con antecedentes de ceguera total postraumática y dificultad para la marcha sin apoyo que no había sido estudiada.

Ingresó en el servicio de cirugía vascular de nuestro centro por presentar isquemia crónica de miembro inferior derecho grado IV, pendiente de tratamiento quirúrgico mediante la amputación supracondílea de dicho miembro. 

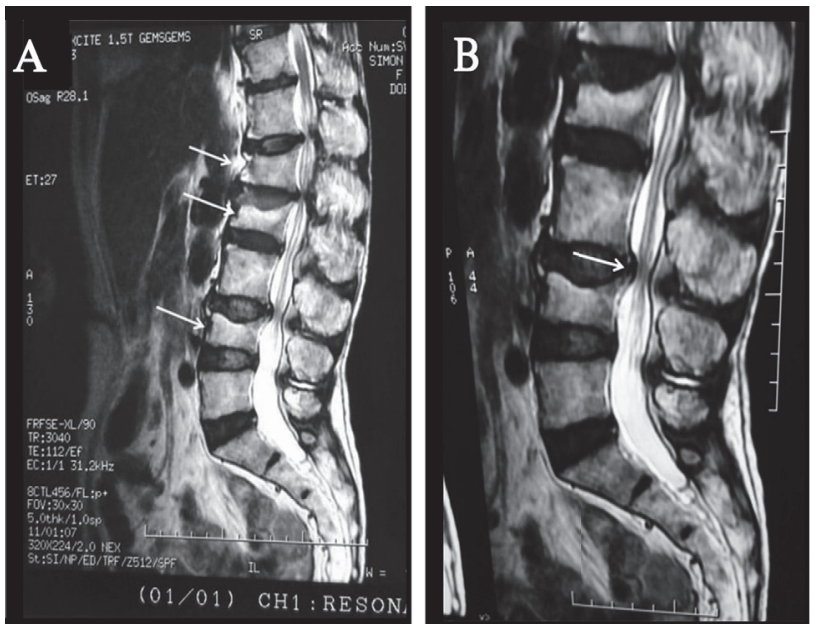

Fig. 1. En las imágenes de esta figura se aprecian las fracturas vertebrales a nivel de L1, L2 y L4 en la imagen A y la estenosis del canal lumbar en la imagen B.

El estudio preoperatorio mostró: anemia microcítica - hipocrómica, coagulación y bioquímica normales y un trastorno de la repolarización en el electrocardiograma.

Durante su estancia en dicho servicio mantuvo dolor del miembro inferior derecho, continuo y progresivamente intolerable, por lo que precisó la colocación de un catéter epidural a nivel de L3-L4 para la conexión de una bomba de infusión PCA (Analgesia Controlada por el Paciente) y administración de una solución compuesta por ropivacaína $0,2 \%$ más cloruro mórfico $0,004 \%$ a una velocidad de $4 \mathrm{ml} / \mathrm{h}$ y bolos de rescate de $4 \mathrm{ml}$. La punción se realizó con una aguja Tuohy 18 G. La colocación del catéter fue técnicamente sencilla y sin incidencias. No hubo aspiración de sangre o de líquido cefalorraquídeo y la dosis test ( $4 \mathrm{ml}$ de bupivacaína $0,25 \%$ con vasoconstrictor) fue negativa. La paciente no había recibido tratamiento anticoagulante durante las 12 horas previas a la colocación del catéter. Tras las primeras 24 horas de la colocación del catéter epidural la paciente presentó un empeoramiento de los síntomas preexistentes: mayor severidad del dolor del miembro inferior derecho, asociado a un bloqueo motor completo de ambos miembros inferiores. Se procedió a suspender la perfusión a través del catéter epidural y se reevaluó a la paciente pasadas 2, 4 y 8 horas.

La exploración a las 8 horas mostró una monoplejía del miembro inferior derecho y una monoparesia del miembro inferior izquierdo, sin incontinencia del esfínter anal (la paciente era portadora de una sonda vesical). En todo momento, nuestra paciente negó la presencia de dolor lumbar.

Se procedió a la retirada del catéter epidural solicitándose un estudio de imagen con RM de columna lumbar en el que se evidenciaron fracturas vertebrales L1, L2 y L4

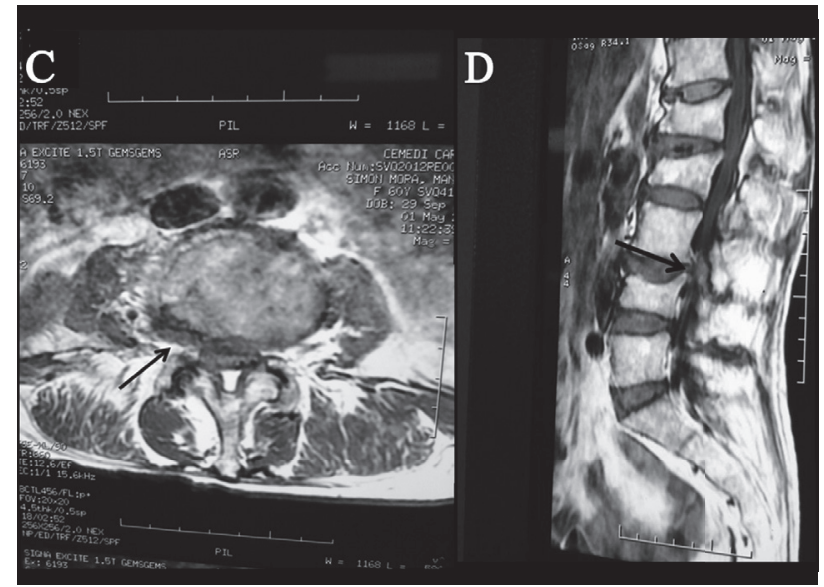

Fig. 2. En las imágenes $\mathrm{C}$ y D se observan, respectivamente, protrusión discal L3-L4 que condiciona compromiso radicular (más marcado en el lado derecho) y un hematoma epidural de pequeño tamaño a nivel L3-L4.

y protrusión discal L3-L4 que condicionaba compromiso radicular (más marcado en el lado derecho) e importante estenosis del canal lumbar (Figs. 1 y 2), agravada por un hematoma epidural de pequeño tamaño a dicho nivel (Fig. 2).

Se inició tratamiento con dexametasona $4 \mathrm{mg} / 6 \mathrm{~h}$, y se procedió a su traslado al servicio de neurocirugía para su valoración.

Durante su ingreso en el servicio de neurocirugía se realizó electromiografía, objetivándose una afectación del sistema nervioso periférico que podría corresponderse con una polineuropatía mixta.

Con todos estos datos, se consideró que la clínica de la paciente era el resultado de una conjunción de múltiples patologías, desestimándose la intervención quirúrgica dado que no se beneficiaría de dicho tratamiento.

Finalmente, la paciente fue trasladada al servicio de cirugía vascular para completar el tratamiento quirúrgico. La amputación supracondílea del miembro inferior derecho se realizó bajo anestesia general con mascarilla laríngea i-gel ${ }^{\mathrm{TM}}$ (Intersurgical Ltd., Wokingham, England) número 4. La inducción de la anestesia se realizó mediante la administración intravenosa de fentanilo $2 \mu \mathrm{g} / \mathrm{kg}$ y propofol $2 \mathrm{mg} / \mathrm{kg}$. El mantenimiento de la misma se realizó con sevofluorano $2 \%$ en una mezcla de oxígeno - aire con un flujo de gas fresco de 2 1/min. La administración adicional de fentanilo durante el procedimiento quirúrgico se realizó según parámetros clínicos, cifras de tensión arterial y frecuencia cardiaca. Se realizó una monitorización estándar de la paciente constatándose estabilidad hemodinámica durante toda la cirugía. Al finalizar el procedimiento quirúrgico se retiró la mascarilla laríngea cuando la paciente estuvo completamente despierta. Fue trasladada a la Unidad de Recuperación Postanestésica 
donde permaneció durante 2 horas. Su evolución fue favorable.

\section{DISCUSIÓN}

Se estima que la incidencia de complicaciones neurológicas tras la anestesia epidural es de 1/1.000-1/1.000.000 (4). Sus principales causas son: absceso epidural o subdural $(0,04-0,07 \%)$, hematoma espinal $(0,04-0,05 \%)$, lesión traumática (con aguja o catéter) y toxicidad directa de los fármacos administrados (5).

El hematoma epidural es, probablemente, la complicación más conocida de la anestesia epidural y fue nuestra primera sospecha en esta paciente. Los síntomas suelen ser los de una compresión medular subaguda: lumbalgia y paraplejía de evolución progresiva. Sin embargo, en nuestra paciente el dolor lumbar estuvo ausente. La mayor severidad del dolor del miembro inferior derecho, asociado a un bloqueo sensitivo-motor completo de ambos miembros inferiores nos hizo pensar que se tratara de un dolor neuropático de origen central.

Estudios previos en los que se ha investigado el riesgo de complicaciones neurológicas asociadas a la anestesia/ analgesia neuroaxial en pacientes con esclerosis múltiple, poliomielitis (6) o neuropatía diabética (7) concluyeron que el riesgo de lesiones neurológicas graves en estos pacientes es poco frecuente. Aún así, dicho riesgo parece ser mayor que el reportado para la población general (8).

Un número creciente de informes, que describen casos similares al nuestro, sugieren que la estenosis espinal no diagnosticada puede ser un importante contribuyente de las complicaciones neurológicas tras realizar un bloqueo neuroaxial $(4,8,9)$.

Un estudio previo refiere una frecuencia de complicaciones neurológicas significativamente superior en los pacientes con diagnóstico preoperatorio de radiculopatía compresiva, estenosis espinal y neuropatía periférica frente a otros diagnósticos neurológicos (10), patologías que fueron diagnosticadas en nuestra paciente tras la retirada del catéter epidural. Este estudio ha sido el primero en caracterizar la frecuencia y la severidad de eventos neurológicos tras el bloqueo neuroaxial en pacientes con diagnóstico previo de estenosis espinal o de enfermedad del disco lumbar, y sus principales hallazgos sugieren que estos pacientes presentan mayor riesgo de complicaciones neurológicas en comparación con la población general debido al desarrollo de nuevos déficits neurológicos o al empeoramiento de los ya existentes $(7,8)$. Por todo esto, la anestesia/analgesia neuroaxial suele considerarse una mala opción en pacientes con estenosis del canal o enfermedad del disco (con o sin cirugía previa de columna).

Son varios los mecanismos propuestos para explicar las complicaciones neurológicas tras un bloqueo neuroaxial: alteración del flujo sanguíneo e isquemia $(4,11,12)$, traumatismo con la aguja o el catéter $(9,13)$, toxicidad directa de los anestésicos locales $(8,14)$ o una combinación de varios de estos mecanismos. Los efectos neurotóxicos pueden verse realzados por la mala distribución de los fármacos debido a los cambios degenerativos de la columna vertebral. También se ha propuesto como mecanismo de daño neurológico la isquemia de la médula espinal debido a la alteración de la microcirculación $(4,15)$.

Para evitar que la técnica anestésica sea señalada como el factor etiológico independiente de la complicación neurológica, es muy importante que el anestesiólogo, antes de decidir la aplicación del bloqueo neuroaxial, obtenga del paciente una historia clínica completa en la que se recabe información sobre los factores predisponentes, como trastornos de la coagulación, tratamiento anticoagulante, infecciones y enfermedades neurológicas previas.

Nuestra paciente fue sometida a una analgesia epidural a nivel L3-L4, coincidiendo con el nivel de su patología raquídea. Su deterioro funcional, tras la colocación del catéter epidural, sugiere un efecto aditivo de múltiples condiciones neurológicas preexistentes (estenosis del canal lumbar, radiculopatía y neuropatía periférica). Los mecanismos fisiopatológicos que creemos precipitaron los síntomas neurológicos fueron: factor mecánico (hematoma epidural de pequeño tamaño), isquemia neuronal a partir de los efectos del volumen administrado por el catéter y posible toxicidad directa del anestésico local $(8,11,16)$.

\section{CONCLUSIÓN}

Los síntomas sospechosos de déficit neurológico, como parestesias o disestesias dolorosas, debilidad motora o trastornos de la marcha, nos obligan a realizar una valoración neurológica exhaustiva del paciente antes de decidir la realización de una técnica neuroaxial.

CORRESPONDENCIA:

Teresa García Navia

Departamento de Anestesiología y Reanimación

Hospital Universitario de Valme

Ctra. de Cádiz Km. 548,9

41014. Sevilla

e-mail.gjusset@yahoo.es

\section{BIBLIOGRAFÍA}

1. Gordh R, Mostert JW. The neurological sequelae of anesthesia (cases 48-60). Int Anesthesiol Clin. 1975;16:115-85.

2. Wulf H. Epidural anesthesia and spinal haematoma. Can J Anaesth. 1996;42:1260-73. 
3. Adamson DC, Bulsara K, Bronec PR. Spontaneous cervical epidural hematoma: case report and literature review. Surg Neurol. 2004;62:156-9; discussion 159-60.

4. De Seze MP, Sztark F, et al. Sever and long lasting complications of the nerve root and spinal cord after central neuraxial blockade. Anesth Analg. 2007;104:975-9.

5. Brull R, Colin J, et al. Neurological complications after regional anesthesia: comtemporary estimates of risk. Anesth Analg. 2007;104:965-74.

6. Hebl JR, Horlocker TT, Schroeder DR. Neuraxial anesthesia and analgesia in patients with preexisting central nervous system disorders. Anesth Analg. 2006;103:223-8.

7. Hebl JR, Kopp SL, Schroeder DR, et al. Neurologic complications after neuraxial anesthesia or analgesia in patients with preexisting peripheral sensorimotor neuropathy or diabetic polyneuropathy. Anesth Analg. 2006;103:1294-9.

8. Moen V, Dahlgren N, Irestedt L. Severe neurological complications after central neuraxial blockades in Sweden 19901999. Anesthesiology. 2004;101:950-9.

9. Stambough JL, Stambough JB, Evans S. Acute cauda equina syndrome after total knee arthroplasty as a result of epidural anesthesia and spinal stenosis. J Arthroplasty. 2000;15: 375-9.
10. Hebl JR, Horlocker TT, Kopp SL, et al. Neuraxial Blockade in Patients with Preexisting Spinal Stenosis, Lumbar Disk Disease, or Prior Spine Surgery: Efficacy and Neurologic Complications. Anesth Analg. 2010;111:1511-9.

11. Usubiaga JE, Wikinski JA, Usubiaga LE. Epidural pressure and its relation to spread of anesthetic solutions in epidural space. Anesth Analg. 1967;46:440-6.

12. Hooten WM, Hogan MS, Sanemann TC, et al. Acute spinal pain during an attempted lumbar epidural blood patch in congenital lumbar spinal stenosis and epidural lipomatosis. Pain Physician. 2008;11:87-90.

13. Tetzlaff JE, Dilger JA, Wu C, et al. Influence of lumbar spine pathology on the incidence of paresthesia during spinal anesthesia. Reg Anesth Pain Med. 1998;23:560-3.

14. Yuen EC, Layzer RB, Weitz SR, et al. Neurologic complications of lumbar epidural anesthesia and analgesia. Neurology. 1995;45:1795-801.

15. Jacob AK, Borowiec JC, Long TR, et al. Transient profound neurologic deficit associated with thoracic epidural analgesia in an elderly patient. Anesthesiology. 2004;101:1470-1.

16. Markman JD, Gaud KG. Lumbar spinal stenosis in older adults: current understanding and future directions. Clin $\mathrm{Ge}-$ riatr Med. 2008;24:369-88. 